\title{
Stress-Induced Despair Behavior Develops Independently of the AHR-RORgt Axis in CD4+ cells
}

\section{Courtney R. Rivet-Noor}

Center for Brain Immunology and Glia

Andrea R. Merchak

Center for Brain Immunology and Glia

Sihan Li

Department of Neuroscience

Rebecca M. Beiter

Center for Brain Immunology and Glia

\section{Sangwoo Lee}

University of Virginia School of Medicine

Jalon Aaron Thomas

University of Virginia School of Medicine

Anthony Fernández-Castañeda

Center for Brain Immunology and Glia

Jung-Bum Shin

Department of Neuroscience

Alban Gaultier ( $\nabla$ ag7h@virginia.edu )

Center for Brain Immunology and Glia

\section{Research Article}

Keywords: approaches, depression, stress

Posted Date: January 3rd, 2022

DOI: https://doi.org/10.21203/rs.3.rs-1181536/v1

License: (c) (1) This work is licensed under a Creative Commons Attribution 4.0 International License.

Read Full License 
Stress-Induced Despair Behavior Develops Independently of the AHR-RORgt Axis in CD4+ cells

Courtney R. Rivet-Noor ${ }^{1,2,3, \$}$, Andrea R. Merchak ${ }^{1,2,3, \$}$, Sihan $\mathrm{Li}^{2,4}$, Rebecca M. Beiter ${ }^{1,2,3}$, Sangwoo Lee $^{5}$, Jalon Aaron Thomas ${ }^{5}$, Anthony Fernández-Castañeda ${ }^{1,2,3}$, Jung-Bum Shin ${ }^{2,4}$ and Alban Gaultier ${ }^{1,2,3, \# \text {. }}$

${ }^{1}$ Center for Brain Immunology and Glia, ${ }^{2}$ Department of Neuroscience, ${ }^{3}$ Graduate Program in Neuroscience, ${ }^{4}$ Graduate program in Biochemistry and Molecular Genetics, ${ }^{5}$ Department of Computer Science, University of Virginia School of Medicine, Charlottesville, VA 22908, USA.

\$C.R.N. and A.R.M. contributed equally to this work, "Corresponding author. Email: ag7h@virginia.edu.

Running Title: Depression Develops without Th17 Cells. 


\begin{abstract}
:
Current treatments for major depressive disorder are limited to neuropharmacological approaches and are ineffective for large numbers of patients. Recently, alternative means have been explored to understand the etiology of depression. Specifically, changes in the microbiome and immune system have been observed in both clinical settings and in mouse models. As such, microbial supplements and probiotics have become a target for potential therapeutics. A current hypothesis for the mechanism of action of these supplements is via the aryl hydrocarbon receptor's (AHR) modulation of the T helper 17 cell (Th17) and T regulatory cell axis. As inflammatory ROR $\mathrm{t}+\mathrm{CD} 4+\mathrm{Th} 17 \mathrm{~T}$ cells and their primary cytokine IL-17 have been implicated in the development of stress-induced depression, the connection between stress, the AHR, Th17s and depression remains critical to disease understanding. Here, we utilize genetic knockouts to examine the role of the microbial sensor AHR in the development of stress induced despair behavior. We observe an AHR-independent increase in gut-associated Th17s in stressed mice, indicating that AHR is not responsible for this communication. Further, we utilized a CD4-specific Rorc knockout line to disrupt the production of Th17s. Mice lacking Rorc induced IL-17 did not show any differences in behavior from controls before or after stress. Finally, we utilize an unsupervised machine learning system to examine minute differences in behavior that could not be observed in traditional behavioral assays. Our data demonstrate that neither CD4 specific Ahr nor Rorc are necessary for the development of stress-induced anxiety- or depressive-like behaviors. These data suggest that research approaches should focus on other sources or sites of IL-17 production in stress-induced depression.
\end{abstract}




\section{Introduction:}

In the United States, an estimated 17.3 million adults are diagnosed with major depressive disorder (MDD), with over $60 \%$ struggling with severe impairments, making MDD the number one cause of disability in the U.S. ${ }^{1}$. Dogma states that depression and other mood disorders are caused by an imbalance in neurotransmitters ${ }^{2-4}$. As such, the majority of existing treatments target neurotransmitter uptake (SSRIs, SNRIs, etc) ${ }^{5}$. However, a significant number of patients do not benefit from these therapeutics, suggesting alternate sources for MDD ${ }^{5}$.

The microbiome has emerged as an important contributor to many neurological conditions, ranging from Parkinson's disease to autism spectrum disorder ${ }^{6,7}$. Thus, it is unsurprising that the microbiome has also been found to play a crucial role in the development and maintenance of MDD. Patients with depression have altered microbiomes when compared to control patients ${ }^{8}$. Microbiome dysbiosis is also present in animal models of MDD $^{9-12}$ and therapeutic administration of probiotics has been found to be beneficial ${ }^{12}$. The mechanism by which the microbiome contributes to the etiology of depression remains under investigation. However, one postulated route is through the immune system ${ }^{13}$.

While there are many ways in which inflammation and the microbiome can interact in depression, a microbiome-responsive regulator of the immune system called the Aryl Hydrocarbon Receptor (AHR) has the potential to serve as a lynchpin in this pathway. Indeed, metabolomics, GWAS, and epigenetic studies have indicated that dysfunction of the AHR is associated with both MDD and post traumatic stress disorder ${ }^{14,15}$. The AHR is a cytoplasmic receptor that can bind microbiome produced metabolites, including tryptophan derived metabolites ${ }^{13}$. AHR activation induces transcription factor activity that can modulate the inflammatory environment of the gut. In particular, AHR has been shown to regulate T helper cell 17 (Th17) and T regulatory cell (Treg) function in response to microbial metabolites ${ }^{16}$. This represents an important connection between the microbiome and immune system as IL-17 produced by ROR $\gamma \mathrm{T}+\mathrm{Th} 17 \mathrm{~s}$ have been shown to contribute to depression- and anxiety-like behaviors ${ }^{17,18}$. Studies have shown that the number of Th17s in the gut associated lymphoid tissue (GALT) increases in response to stress and correlates with depressive-like behavior ${ }^{19}$. Additionally, the transfer of Th17 cells into mice has been found to induce depressive-like behaviors ${ }^{17}$. Administration of IL-17A blocking antibodies has also been found to reduce learned helplessness behaviors in mice ${ }^{17}$. However, the evidence for the role of IL-17 in human depression remains controversial, with some reports claiming a positive correlation between the inflammatory cytokine and depression and others reporting no differences in IL-17 levels between those with the disease and controls ${ }^{20-25}$. These conflicting results highlight the need for further investigation into the mechanism and role of the AHR and increased inflammatory Th17 cells in depression.

Here, for the first time, we use genetic tools and artificial intelligence behavior analysis to assess the contribution of T cell specific AHR and IL-17 to the development of anxiety- and depressive-like behaviors in a murine model of stress. While our data confim that stressed mice present with a larger number of Th17 cells in the gut, our behavioral analyses show that the deletion of either AHR or ROR $\gamma \mathrm{T}$ in T cells does not impact anxiety- or depressive-like behaviors in mice. Taken together our data suggest that ROR $\gamma \mathrm{T}$ induced IL-17 in T cells is not necessary for the pathological development of depressive-like behaviors induced by unpredicalble chronic mild restraint stress. 


\section{Materials and Methods:}

All methods were performed in accordance with guidelines and regulations of the University of Virginia and approved by the University of Virginia Animal Care and Use Committee. Experiments involving live animals are reported as described by the ARRIVE Guidelines.

Mice:

Cd4 Cre $^{26}$ (\#022071), Rorc ${ }^{\mathrm{F} / \mathrm{F}} 18$ (\#008771), and Ahr ${ }^{\mathrm{F} / \mathrm{F} 27}$ mice were purchased from Jackson Laboratories. Mice were bred in house. Mice were kept on a 12 hour light/dark schedule. All behavioral interventions were performed between 8am and 3pm and animals were sacrificed between $7 \mathrm{am}$ and $1 \mathrm{pm}$. All mice were housed in cages of up to 5 animals from birth until initiation of the stress protocol. All mice exposed to stress were at least 8 weeks of age and age matched to control animals. Stressed mice were housed individually without enrichment to enhance stress $^{28}$. Naïve animals were housed in standard cages in groups of 2-5 mice of the same sex. All procedures were approved by the University of Virginia ACUC (protocol \#1918).

Experimental Autoimmune Encephalomyelitis:

EAE was induced in 6-8-week old female Cd4 Cre $A h r^{F / F}$ and $A h r^{F / F}$ mice as previously described ${ }^{29}$. Thirty-minute videos of mice were taken at day 25 post immunization and used for analysis. Healthy controls were age matched females.

\section{Stress Experiments:}

In all Unpredictable Chronic Mild Restraint Stress (UCMRS) experiments, mice were exposed to chronic restraint (ventilated $50 \mathrm{~mL}$ conical vials) for a period of 2 hours daily. Once removed from restraint, an overnight stressor of either cage tilt, wet bedding, or $2 x$ cage change was used. All daily stressors were carried out between $8 \mathrm{am}$ and $5 \mathrm{pm}$. Overnight stressors were started upon removal from the daily stressor and remained in place until the next day's daily stressor.

\section{Behavioral Tests:}

The forced swim, tail suspension, sucrose preference, open field, elevated plus maze, novel object recognition, marble burying, and three chamber social preference tests we performed as previously described ${ }^{7,30-36}$. All testing was recorded on a Hero Session 5 GoPro and analyzed with Noldus behavioral analysis software.

\section{DeepLabCut:}

Animal pose estimation: Animal pose estimation was performed by using a deep-learning package, DeepLabCut ${ }^{37}$ (https://github.com/DeepLabCut/DeepLabCut). We generated a DeepLabCut convolutional neural network to analyze our open field test videos, which is trained in a supervised manner: 16 manually labeled points were selected as references of transfer learning. 15 randomly selected videos were used for building a training dataset. Finally, the performance of the neural network is evaluated by researchers.

Unsupervised behavior classification: Estimated mouse poses from DeepLabCut were further analyzed by Variational Animal Motion Embedding $(\mathrm{VAME})^{38}$, which classifies animal behavior in an unsupervised manner (https://github.com/LINCellularNeuroscience/VAME). We trained a 
unique VAME recursive neural network for each experiment, which classifies each frame of the open field test video into 1 of the 35 behavioral motifs. Then, all behavior motifs were annotated and evaluated by blinded researchers. With annotated frames, we were able to calculate the percentage of time usage of each motif, which is then used for principal component analysis and Kullback-Leibler divergence analysis.

RNA Extraction and Quantitative PCR:

For RNA extraction, cultured cells were pelleted, frozen, lysed, and RNA extracted using the Bioline Isolate II RNA mini kit as per manufacture's protocol (BIO-52073). RNA was quantified with a Biotek Epoch Microplate Spectrophotometer. Normalized RNA was reverse transcribed to cDNA with either the Bioline SensiFast cDNA Synthesis Kit (BIO-65054) or Applied Sciences HighCapacity cDNA Reverse Transcriptase Kit (43-688-13). cDNA was amplified using the Bioline SensiFast NO-ROX kit (BIO-86020), according to manufacturer's instructions. The TaqMan GAPDH probe (Mm99999915_g1) was measured as a normalizer for each sample. The TaqMan probes Cyp1a1 (Mm00487218_m1), Ahr (Mm00478932_m1), Rorc (Mm01261019_g1), and Il17a (Mm00439618_m1) were used to measure transcript levels from the samples. Results were analyzed with the relative quantity $(\Delta \Delta \mathrm{Cq})$ method.

CD4 T cells isolation and differentiation:

Naïve CD4 T cells were harvested and skewed as previously described ${ }^{39}$. After skewing, cells were washed and frozen for qPCR analysis or treated with an AHR antagonist $\left(I_{3} S-250 u M\right.$, SigmaAldrich I3875-1G), agonist (CH223191-10uM, Tocris Bioscience 301326-22-7), or vehicle control (DMSO-Fisher Scientific D128-1) for 24 hours prior to freezing.

Tissue Harvest and Digestion:

After experimental manipulation, mice were perfused with $0.9 \%$ saline plus 5 units $/ \mathrm{mL}$ heparin (Medefil; MIH-3333) and tissues of interest were harvested and processed for flow cytometry as described below.

Small Intestine:

Whole small intestine was collected from the animals, flayed open and rinsed with ice cold HBSS (Gibco, 14175-095). Tissue was cut into $2 \mathrm{~cm}$ pieces and stored in $30 \mathrm{~mL}$ of $5 \%$ FBS (R\&D systems, $\mathrm{S} 12450 \mathrm{H}$ ) in $\mathrm{HBSS}$ until processing. Small intestine was shaken at $37^{\circ} \mathrm{C}$ for 20 minutes to remove mucus and debris. Gut pieces were filtered over mosquito net, placed in fresh $30 \mathrm{~mL}$ of $5 \%$ FBS in HBSS, and shaken at $37^{\circ} \mathrm{C}$ for another 20 minutes. Samples were again filtered over mosquito net. Pieces were cut using a razor blade until fine slurry was created. Slurry was incubated in gut digestion buffer: Collagenase 8 (Sigma, C2139-5G), DNAse (Worthington, LS002139) in 5\% FBS in HBSS-/- for $40 \mathrm{~min}$, shaken at $150 \mathrm{rpm}$ at $37^{\circ} \mathrm{C}$. Once digested, the solution was filtered through a $70 \mu \mathrm{m}$ filter and washed three times with $5 \%$ FBS in HBSS.

Lymph nodes and Peyer's Patches:

Single cell suspension in RPMI was prepared from Peyer's Patches and lymph nodes after fat removal by mechanical dissociation and subsequent filtration using sterile $70 \mu \mathrm{m}$ filters. 
Meninges:

Meninges were dissected from skull caps in ice cold RPMI and digested in the digestion buffer: Collagenase 2 (Gibco, 17101-015), collagenase D (Sigma, 11088882001) and DNAse (Worthington, LS002139) for 20 minutes at $37^{\circ} \mathrm{C}$. Once digested, meninges were physically dissociated with a $1 \mathrm{~mL}$ pipette and filtered through a sterile $70 \mu \mathrm{m}$ filter.

Flow cytometry

Single cell suspensions were incubated with CD16/32 Fc Block and then stained with a 1:200 antibody dilution (1:100 for transcription factors). For intranuclear staining, the eBioscience FoxP3/Transcription Factor Staining Kit (00-5523-00) was used per manufacturer's instructions. Antibodies used are as follows: 488-conjugated CD8 (53-008182), APCe780-conjugated TCR (475961-82), e450-conjugated CD4 (48-0042-820, PE-conjugated ROR $\gamma^{\top}$ (12-6981-82), and PE-Cy7conjugated FoxP3 (25-5773-82), all purchased from Invitrogen. APC-conjugated CD45.2 (109813) was purchased from BioLegend. A Live/Dead discrimination dye Ghost Dye Violet 510 (Tonbo Biosciences; 13-0870) was used on all samples. OneComp eBeads (Thermo Fisher Scientific, 01111-42) were used for all color controls except for the viability dye in which cells were used. Flow cytometry was performed using a Beckman Coulter Gallios flow cytometer and data were analyzed with FlowJo software v10.7.1.

Statistical Analysis:

All statistical analyses-except those associated with DeepLabCut-were performed in GraphPad Prism 9. Analyses involving two groups were performed using a two-tailed t-test. If the variances between groups were significantly different, a Welch's correction was applied. Outliers were excluded if they fell more than two standard deviations from the mean. For all analyses, the threshold for significance was at $p<0.05$. Repeats for each experiment are specified in the figure legend corresponding to the respective panel.

\section{Results:}

\section{Absence of AHR in T cells does not Influence Baseline Behaviors:}

We and others have previously shown that microbiome dysbiosis induced by unpredictable chronic mild stress (UCMS) is a contributing factor to depressive- and anxiety-like behaviors in mice ${ }^{12,40,41}$. Furthermore, therapeutic reconstitution with lactobacillus is sufficient to correct behaviors elucidated by $\mathrm{UCMS}^{12}$. Lactobacillus is known to produce metabolites that engage the AHR receptor, such as tryptophan metabolites which can modify the immune environment ${ }^{42}$. Given the emerging role of $T$ cells and their cytokines in depression ${ }^{17,43,44}$, we wanted to determine whether T cell AHR sensing of the microbiome leads to immunological and behavioral changes associated with stress. We generated $C d 4 C r e ~ A h r^{\text {flox/flox }}$ mice $(A h r \mathrm{KO})$ to examine depressive- and anxiety-like behaviors in the absence of this important receptor in the $T$ cell compartment. As expected, $A$ hr expression was significantly reduced in CD4+ T cells isolated from Ahr KO mice by qPCR (Fig. 1A). Furthermore, in vitro generated Th17 cells prepared from Ahr KO animals failed to upregulate the AHR downstream transcription factor Cyp1b1 in response to AHR activation using idoxyl-3-sulfate (I3S) ${ }^{45}$. Ahr KO levels of Cyp1b1 expression were consistent with those of AHR competent cells treated with an AHR antagonist $\mathrm{CH} 223191$. This confirmed a robust 
functional deletion of AHR in T cells (Fig. 1B). Next, we explored whether lacking AHR in T cells could influence behaviors at baseline. Both depressive- and anxiety-like behavioral assays were performed on Ahr KO mice and age matched controls. No differences were observed in the forced swim, tail suspension, or nestlet shredding tests (Fig. 1C). These results show that mice lacking AHR in T cells do not present with baseline depressive- or anxiety-like behaviors.

\section{UCMRS Drives a Specific Expansion of Th17 cells in the Lamina Propria in an AHR-Independent} Manner:

To test the role of the T cell specific AHR in stress response, we subjected Ahr KO or age matched controls to 3 weeks of UCMRS (Fig. 2A). Surprisingly, no differences in anxiety- or depressive-like behaviors were observed between groups after stress (Fig. 2B). While classic assays may detect strong phenotypic differences, they may not detect more subtle behavioral changes. To circumvent this limitation, we applied an unsupervised machine learning approach to analyze behaviors in both genotypes using DeepLabCut ${ }^{37}$. First, we validated this computational approach using a preclinical model of multiple sclerosis (MS) known as experimental autoimmune encephalomyelitis (EAE). EAE produces significant locomotor changes and should produce detectable motor differences between groups to act as a positive control. DeepLabCut was able to detect robust behavioral differences between groups in nearly $40 \%$ of behavioral motifs characterized by the software (Sup Fig.1 A-F). However, no differences were found between stressed, Ahr KO animals and age matched controls as shown by Kullback-Leibler Divergence-a measure quantifying variances within and between groups-(Fig. 2C and Sup Fig. 2B) and by PCA (Fig. 2D). Similarly, of all behavioral motifs analyzed, only one showed a significant change between groups ( $2.8 \%$ of all behaviors), suggesting the overall behaviors of $A h r$ KO mice are very similar to control animals in response to stress (Sup Fig. 2C-D). Additionally, immunophenotyping revealed no differences in the measured immune compartment between Ahr KO and control mice (Sup Fig. 2A). Strikingly, however, both the Ahr KO animals and controls had an increase in the number of CD4+ROR $\gamma \mathrm{T}+$ Th17 cells in the GALT after stress (Fig. 2E). Thus, the microbiome changes and increase in Th17 cells observed in response to stress is not mechanistically linked by AHR activation in CD4+ cells.

Deletion of Rorc in T cells does not Induce Spontaneous Anxiety- or Depressive-like Behaviors: To explore the role Th17 cells in anxiety- and depressive-like behaviors, Cd4 Cre Rorc flox/flox mice were generated (Rorc KO). Knock down of ROR $\gamma T$ was confirmed using in vitro derived Th17s by examining levels of I/17 and Rorc by qPCR (Fig. 3A) and IL-17 secretion by ELISA quantification (Fig. 3B). Immunophenotyping on spleen revealed the absence of CD4+ROR $\gamma T+T$ cells in vivo (Sup. Fig. 3A-C). Baseline behaviors were compared between male Rorc KO mice and controls. No differences between the Rorc KO mice or age matched controls were observed in tasks used to assess depressive- (Fig. 3D) or anxiety- like behaviors (Fig. 3F). As Th17 cells are also known to contribute to autism-like behaviors in male mice ${ }^{18}$, we analyzed the marble burying (Fig. $3 \mathrm{C}$ ), social preference (Fig. 3E) and novel object recognition (Fig. 3G) tests at baseline. No differences between genotypes were observed in these behavior tests. Lastly, to ensure there were no subtle behavior differences between groups that could not be quantified with known behavioral tasks, we again used machine learning to examine unbiased behavioral clusters. No differences were found in the Kullback-Leibler Divergence plot (Fig. 3H and Sup Fig. 3D) and a large overlap in the 
PCA plot was observed (Fig. 3I). Additionally, only 3 of 35 behavioral motifs demonstrated significant differences between groups after DeepLabCut analysis (Sup. Fig. 3C-F). Together, these data suggest that at baseline, a lack of Rorc from development does not impact autism-, depression-, or anxiety-like behaviors in male mice.

\section{UCMRS Induced Anxiety- or Depressive-like Behaviors are not Affected by the Lack of Rorc in Th17 cells:}

While no behavioral differences in male mice were observed between groups at baseline, females experience depression at a higher rate than males ${ }^{46}$. Thus, we aimed to examine the impact of Rorc in Th17 cells in female mice. Like the male mice, no differences in escape behavior, anhedonia, or anxiety-like behaviors were observed at baseline (Fig. 4A-B). Similarly, our machine learning approach was not able to detect differences in behaviors as shown through the KullbackLeibler Divergence plot (Fig. 4C and Sup. Fig. 4C), PCA plot (Fig. 4D), and motif usage breakdown plots (Sup. Fig. 4A-B). To examine the impacts of Rorc KO in a stressful environment, female mice were exposed to 3 weeks of UCMRS. After exposure to stress, no differences between groups were observed in escape behavior (Fig. 4E). The nestlet shred test revealed that Rorc KO mice demonstrate a significant increase in anxiety-like nesting behaviors, whereas the elevated plus maze showed a significant decrease in anxiety-like behaviors, and the open field test showed no difference between groups (Fig. 4F). Ultimately, our unbiased computational approach also did not detect any differences as seen in the Kullback-Leibler Divergence plot (Fig. 4G and Sup. Fig. 4F) and the PCA plot (Fig. 4H). No differences in groups were detected in any of the 35 behavior motifs identified between groups. In summary, although we saw a significant increase in anxietylike behavior in the nestlet shredding test between Rorc KO and control animals, our other behavioral tests did not support this trend and our unbiased behavioral analysis showed no changes between groups. Based on these data, Rorc KO in T cells does not significantly impact depressive-like behaviors in female mice before or after UCMRS.

\section{Discussion:}

Depression presents a major social, health, and economic concern across the world, representing the number one cause of disability ${ }^{47}$. The root causes of this debilitating disorder have yet to be elucidated. With treatments remaining inconsistent and new options lacking, new research is needed to further our understanding of this disorder. Both the microbiome and $T$ lymphocytes have been gaining support as potential mediators of MDD pathology. Here, we examine the role of $T$ cell specific ROR $\gamma T$ and $A H R$ in a mouse model of depression. We demonstrate that deletion of the Ahr gene does not impact anxiety- or depressive-like behaviors at baseline or after chronic stress exposure. Additionally, while we see an increase in the number of ROR $\gamma T+$ cells in the lamina propria of stressed mice, it occurs independently of the AHR. This indicates that there is another mechanism for Th17 cell expansion in response to stress other than environmental sensing through the AHR.

Further, when Rorc is deleted from CD4+ T cells, no changes in anxiety or depressive-like behaviors are observed at baseline or after stress exposure. This is supported by previous work showing that increasing Th17s is not sufficient to change the susceptibility of mice to social defeat stress $^{43}$. Others have suggested that increased Th17s are responsible for stress-induced depressive-like behaviors and have supported this claim by transferring Th17s into mice and 
finding increases in depressive-like behaviors. However, this only suggests that an increase in Th17s may be sufficient to drive these behaviors ${ }^{17}$, not that it is necessary. Additionally, the origin of IL-17 is not precisely defined. IL-17 can be produced from many cell types and be induced in alternative ways that may contribute to depression outside of Th17s ${ }^{48,49}$. Supporting this concept, it has been found that IL-17 from $\gamma \delta$ T cells regulates anxiety-like behaviors in mice ${ }^{44}$. Additionally, multiple transcription factors (STAT3, NF- KB, KLF4, etc) and microRNA can act on IL-17 producing cells to induce IL-17 production ${ }^{50}$. This regulation can act in synergy with ROR $\gamma$ T or independently of it, as with $\mathrm{KLF} 4^{50}$, suggesting that IL-17 could be produced without Rorc and outside of Th17 cells. These data and our own work suggest that if there is a role of IL-17 in the onset of depression, this cytokine does not solely originate from CD4+ cells or is produced by alternative cell type (ILC3s, $\gamma \delta$ T cells, or others).

The notion that Th17 cells play a critical role in the onset of depression has been growing in popularity but remains controversial. Several human studies have demonstrated that changes in IL-17 are correlated with depression ${ }^{21,23,25,51}$ while others have not ${ }^{20,24}$. While further work is needed to fully understand the role of IL-17 in the onset of depressive symptoms, our work demonstrates that the AHR is not responsible for the observed stress-induced increase in intestinal Th17s and that these cells are not necessary for the induction of stress-induced depression. Instead, we suggest there are alternative means or sites of IL-17 production in stressinduced depression. If no such mechanisms can be identified, a need to develop novel approaches to identify other potential causes of depression symptoms, such as the microbiome or other inflammatory signals is required.

\section{Acknowledgements}

The authors are supported by grants from the NIMH R33 MH108156, the UVA Trans University Microbiome Initiative pilot grant, and the Owens Family Foundation. C.R.N is supported by the UVA Presidential Fellowship in Neuroscience. A.R.M. is supported by the NINDS T32 NS115657 J.B.S is supported R01 DC018842.

\section{Contributions}

C.R.N., A.R.M. and A.G. designed the study; C.R.N., A.R.M., A.F.C. and R.M.B. performed experiments; S.L., S.L., J.A.T. and J.B.S performed computational analysis; C.R.N. and A.R.M. analyzed the data and wrote the manuscript; A.G. oversaw the project.

\section{Competing Interests}

Authors declare no competing interests. 


\section{References}

1 Malhi, G. S. \& Mann, J. J. Depression. Lancet (London, England) 392, 2299-2312, doi:10.1016/s0140-6736(18)31948-2 (2018).

2 Mulinari, S. Monoamine theories of depression: historical impact on biomedical research. Journal of the history of the neurosciences 21, 366-392, doi:10.1080/0964704x.2011.623917 (2012).

3 Ban, T. A. Pharmacotherapy of depression: a historical analysis. Journal of neural transmission (Vienna, Austria : 1996) 108, 707-716, doi:10.1007/s007020170047 (2001).

4 Tissot, R. The common pathophysiology of monaminergic psychoses: a new hypothesis. Neuropsychobiology 1, 243-260, doi:10.1159/000117498 (1975).

5 Blier, P. Neurobiology of depression and mechanism of action of depression treatments. The Journal of clinical psychiatry 77, e319, doi:10.4088/JCP.13097tx3c (2016).

6 Sampson, T. R. et al. Gut Microbiota Regulate Motor Deficits and Neuroinflammation in a Model of Parkinson's Disease. Cell 167, 1469-1480.e1412, doi:https://doi.org/10.1016/j.cell.2016.11.018 (2016).

7 Lammert, C. R. et al. Cutting Edge: Critical Roles for Microbiota-Mediated Regulation of the Immune System in a Prenatal Immune Activation Model of Autism. The Journal of Immunology 201, 845-850, doi:10.4049/jimmunol.1701755 (2018).

8 Jiang, H. et al. Altered fecal microbiota composition in patients with major depressive disorder. Brain, behavior, and immunity 48, 186-194, doi:10.1016/j.bbi.2015.03.016 (2015).

$9 \mathrm{Li}, \mathrm{N}$. et al. Fecal microbiota transplantation from chronic unpredictable mild stress mice donors affects anxiety-like and depression-like behavior in recipient mice via the gut microbiota-inflammation-brain axis. Stress 22, 592-602, doi:10.1080/10253890.2019.1617267 (2019).

10 Pearson-Leary, J. et al. The gut microbiome regulates the increases in depressive-type behaviors and in inflammatory processes in the ventral hippocampus of stress vulnerable rats. Molecular Psychiatry, doi:10.1038/s41380-019-0380-x (2019).

11 Zheng, P. et al. Gut microbiome remodeling induces depressive-like behaviors through a pathway mediated by the host's metabolism. Mol Psychiatry 21, 786-796, doi:10.1038/mp.2016.44 (2016).

12 Marin, I. A. et al. Microbiota alteration is associated with the development of stressinduced despair behavior. Scientific reports 7, 43859-43859, doi:10.1038/srep43859 (2017).

13 Merchak, A. \& Gaultier, A. Microbial metabolites and immune regulation: New targets for major depressive disorder. Brain, Behavior, \& Immunity - Health 9, 100169, doi:https://doi.org/10.1016/j.bbih.2020.100169 (2020).

14 Smith, A. K. et al. Epigenome-wide meta-analysis of PTSD across 10 military and civilian cohorts identifies methylation changes in AHRR. Nature Communications 11, 5965, doi:10.1038/s41467-020-19615-x (2020).

15 Liu, D. et al. Beta-defensin 1, aryl hydrocarbon receptor and plasma kynurenine in major depressive disorder: metabolomics-informed genomics. Translational psychiatry 8, 10, doi:10.1038/s41398-017-0056-8 (2018). 
16 Omenetti, S. \& Pizarro, T. T. The Treg/Th17 Axis: A Dynamic Balance Regulated by the Gut Microbiome. Front Immunol 6, 639, doi:10.3389/fimmu.2015.00639 (2015).

17 Beurel, E., Harrington, L. E. \& Jope, R. S. Inflammatory T helper 17 cells promote depression-like behavior in mice. Biol Psychiatry 73, 622-630,

doi:10.1016/j.biopsych.2012.09.021 (2013).

18 Choi, G. B. et al. The maternal interleukin-17a pathway in mice promotes autism-like phenotypes in offspring. Science (New York, N.Y.) 351, 933-939,

doi:10.1126/science.aad0314 (2016).

19 Westfall, S. et al. Microbiota metabolites modulate the Thelper 17 to regulatory T cell (Th17/Treg) imbalance promoting resilience to stress-induced anxiety- and depressivelike behaviors. Brain, behavior, and immunity 91, 350-368, doi:10.1016/j.bbi.2020.10.013 (2021).

20 Saraykar, S. et al. Plasma IL-17A levels in patients with late-life depression. Revista brasileira de psiquiatria (Sao Paulo, Brazil : 1999) 40, 212-215, doi:10.1590/1516-44462017-2299 (2018).

21 Tsuboi, H. et al. Elevated Levels of Serum IL-17A in Community-Dwelling Women with Higher Depressive Symptoms. Behavioral sciences (Basel, Switzerland) 8, doi:10.3390/bs8110102 (2018).

22 Kohler, C. A. et al. Peripheral cytokine and chemokine alterations in depression: a metaanalysis of 82 studies. Acta psychiatrica Scandinavica 135, 373-387, doi:10.1111/acps.12698 (2017).

23 Davami, M. H. et al. Elevated IL-17 and TGF-beta Serum Levels: A Positive Correlation between T-helper 17 Cell-Related Pro-Inflammatory Responses with Major Depressive Disorder. Basic and clinical neuroscience 7, 137-142, doi:10.15412/j.Bcn.03070207 (2016).

24 Kim, J. W. et al. Plasma Levels of IL-23 and IL-17 before and after Antidepressant Treatment in Patients with Major Depressive Disorder. Psychiatry investigation 10, 294299, doi:10.4306/pi.2013.10.3.294 (2013).

25 Liu, Y., Ho, R. C. \& Mak, A. The role of interleukin (IL)-17 in anxiety and depression of patients with rheumatoid arthritis. International journal of rheumatic diseases 15, 183187, doi:10.1111/j.1756-185X.2011.01673.x (2012).

26 Lee, P. P. et al. A critical role for Dnmt1 and DNA methylation in T cell development, function, and survival. Immunity 15, 763-774, doi:10.1016/s1074-7613(01)00227-8 (2001).

27 Walisser, J. A., Glover, E., Pande, K., Liss, A. L. \& Bradfield, C. A. Aryl hydrocarbon receptor-dependent liver development and hepatotoxicity are mediated by different cell types. Proc Natl Acad Sci U S A 102, 17858-17863, doi:10.1073/pnas.0504757102 (2005).

28 Fox, C., Merali, Z. \& Harrison, C. Therapeutic and protective effect of environmental enrichment against psychogenic and neurogenic stress. Behavioural brain research 175, 1-8, doi:https://doi.org/10.1016/j.bbr.2006.08.016 (2006).

29 Fernández-Castañeda, A. et al. The active contribution of OPCs to neuroinflammation is mediated by LRP1. Acta Neuropathol 139, 365-382, doi:10.1007/s00401-019-02073-1 (2020). 
30 Castagne, V., Moser, P., Roux, S. \& Porsolt, R. D. Rodent models of depression: forced swim and tail suspension behavioral despair tests in rats and mice. Current protocols in neuroscience Chapter 8, Unit 8.10A, doi:10.1002/0471142301.ns0810as55 (2011).

31 Liu, M.-Y. et al. Sucrose preference test for measurement of stress-induced anhedonia in mice. Nature Protocols 13, 1686-1698, doi:10.1038/s41596-018-0011-z (2018).

32 Diniz, L., Dos Reis, B. B., de Castro, G. M., Medalha, C. C. \& Viana, M. B. Effects of chronic corticosterone and imipramine administration on panic and anxiety-related responses. Brazilian journal of medical and biological research = Revista brasileira de pesquisas medicas e biologicas 44, 1048-1053 (2011).

33 Cohen, S. J. \& Stackman, R. W., Jr. Assessing rodent hippocampal involvement in the novel object recognition task. A review. Behavioural brain research 285, 105-117, doi:10.1016/j.bbr.2014.08.002 (2015).

34 Ennaceur, A. \& Delacour, J. A new one-trial test for neurobiological studies of memory in rats. 1: Behavioral data. Behavioural brain research 31, 47-59, doi:10.1016/01664328(88)90157-x (1988).

$35 \mathrm{Li}, \mathrm{X} ., \mathrm{Morrow}, \mathrm{D}$. \& Witkin, J. M. Decreases in nestlet shredding of mice by serotonin uptake inhibitors: comparison with marble burying. Life sciences 78, 1933-1939, doi:10.1016/j.Ifs.2005.08.002 (2006).

36 Moy, S. S. et al. Sociability and preference for social novelty in five inbred strains: an approach to assess autistic-like behavior in mice. Genes, Brain and Behavior 3, 287-302, doi:https://doi.org/10.1111/j.1601-1848.2004.00076.x (2004).

37 Mathis, A. et al. DeepLabCut: markerless pose estimation of user-defined body parts with deep learning. Nature Neuroscience 21, 1281-1289, doi:10.1038/s41593-018-0209y (2018).

38 Luxem, K., Fuhrmann, F., Kürsch, J., Remy, S. \& Bauer, P. Identifying Behavioral Structure from Deep Variational Embeddings of Animal Motion. bioRxiv, 2020.2005.2014.095430, doi:10.1101/2020.05.14.095430 (2020).

39 Seki, S. M. et al. Modulation of PKM activity affects the differentiation of $\mathrm{T}<\mathrm{sub}>\mathrm{H}</$ sub $>17$ cells. Science Signaling 13, eaay9217, doi:10.1126/scisignal.aay9217 (2020).

40 Chevalier, G. et al. Effect of gut microbiota on depressive-like behaviors in mice is mediated by the endocannabinoid system. Nature Communications 11, 6363, doi:10.1038/s41467-020-19931-2 (2020).

41 Jianguo, L., Xueyang, J., Cui, W., Changxin, W. \& Xuemei, Q. Altered gut metabolome contributes to depression-like behaviors in rats exposed to chronic unpredictable mild stress. Translational psychiatry 9, 40, doi:10.1038/s41398-019-0391-z (2019).

42 Schiering, C. et al. Feedback control of AHR signalling regulates intestinal immunity. Nature 542, 242-245, doi:10.1038/nature21080 (2017).

43 Ambree, O. et al. Social Defeat Modulates T Helper Cell Percentages in Stress Susceptible and Resilient Mice. International journal of molecular sciences 20, doi:10.3390/ijms20143512 (2019).

$44 \quad$ Alves de Lima, K. et al. Meningeal $\gamma \delta$ T cells regulate anxiety-like behavior via IL-17a signaling in neurons. Nature immunology 21, 1421-1429, doi:10.1038/s41590-020-07764 (2020). 
45 Yang, X. et al. Constitutive regulation of CYP1B1 by the aryl hydrocarbon receptor (AhR) in pre-malignant and malignant mammary tissue. Journal of Cellular Biochemistry 104, 402-417, doi:https://doi.org/10.1002/jcb.21630 (2008).

46 Albert, P. R. Why is depression more prevalent in women? Journal of psychiatry \& neuroscience : JPN 40, 219-221, doi:10.1503/jpn.150205 (2015).

47 Behrens-Wittenberg, E. \& Wedegaertner, F. Identifying Individuals at High Risk for Permanent Disability From Depression and Anxiety. Frontiers in psychiatry 11, doi:10.3389/fpsyt.2020.00740 (2020).

48 McGeachy, M. J., Cua, D. J. \& Gaffen, S. L. The IL-17 Family of Cytokines in Health and Disease. Immunity 50, 892-906, doi:https://doi.org/10.1016/j.immuni.2019.03.021 (2019).

49 Monin, L. \& Gaffen, S. L. Interleukin 17 Family Cytokines: Signaling Mechanisms, Biological Activities, and Therapeutic Implications. Cold Spring Harb Perspect Biol 10, a028522, doi:10.1101/cshperspect.a028522 (2018).

50 Khan, D. \& Ansar Ahmed, S. Regulation of IL-17 in autoimmune diseases by transcriptional factors and microRNAs. Front Genet 6, 236, doi:10.3389/fgene.2015.00236 (2015).

51 Gałecka, M. et al. Inflammatory versus Anti-Inflammatory Profiles in Major Depressive Disorders - The Role of IL-17, IL-21, IL-23, IL-35 and Foxp3. Journal of Personalized Medicine 11, 66 (2021). 


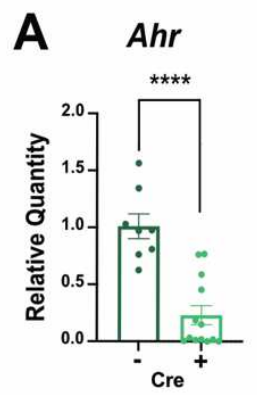

B $\quad$ Cyp1b1
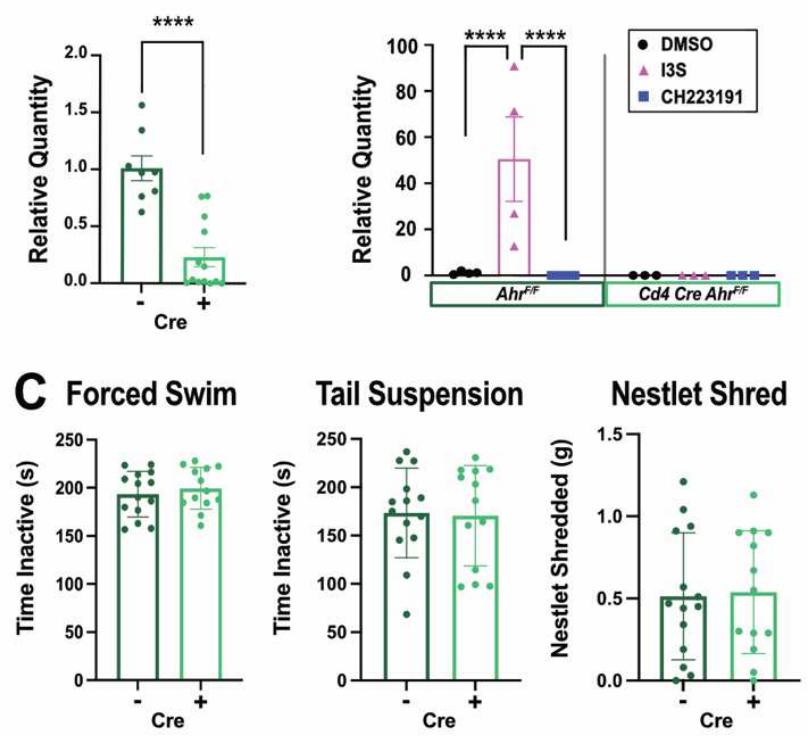

Figure 1: Absence of AHR in T cells does not Influence Baseline Behavior. Relative quantity of Ahr expression from in vitro skewed Th17 cells in Ahr KO and Ahr competent cells (A). T test, each dot represents an animal. Relative quantity of $C y p 1 b 1$ expression from KO and competent cells skewed in vitro to become Th17s and treated with an AHR agonist (I3S), antagonist (CH223191), or control media (DMSO). $\mathrm{N}=2$ (B). One-way ANOVA. Baseline behaviors between Ahr KO and littermate controls (C). T tests, each dot represents an animal, $\mathrm{N}=2$-a single representative experiment shown, male mice. 

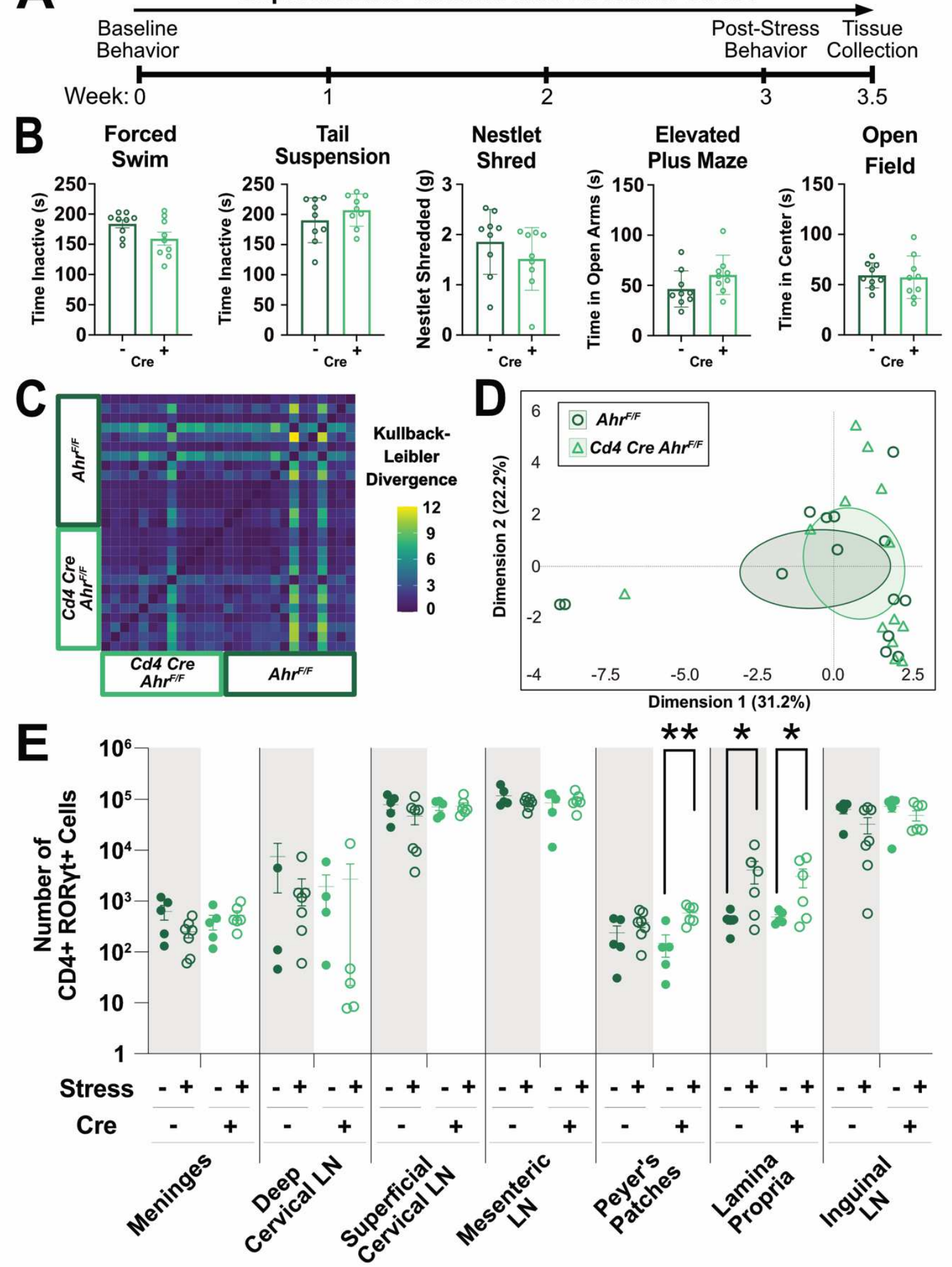

Figure 2: UCMRS Drives Expansion of Th17s in the Lamina Propria in an Ahr Independent Manner. Schematic representing timeline for unpredictable chronic mild restraint stress in mice 
(A). Measures of learned helplessness and anxiety-like behaviors between Ahr KO and littermate controls (B). T tests, $n=9 /$ group. Kullback-Leibler divergence heatmap (C) and PCA plot (D) of DeepLabCut analyzed behaviors between groups. $\mathrm{N}=2$-a single representative experiment shown. Number of $\mathrm{CD} 4+\mathrm{ROR} \gamma \mathrm{T}+$ cells in various immune tissues between stressed and naïve Ahr KO and littermate controls(E). One-way ANOVA, each dot represents an animal, $\mathrm{N}=1$, male mice. 

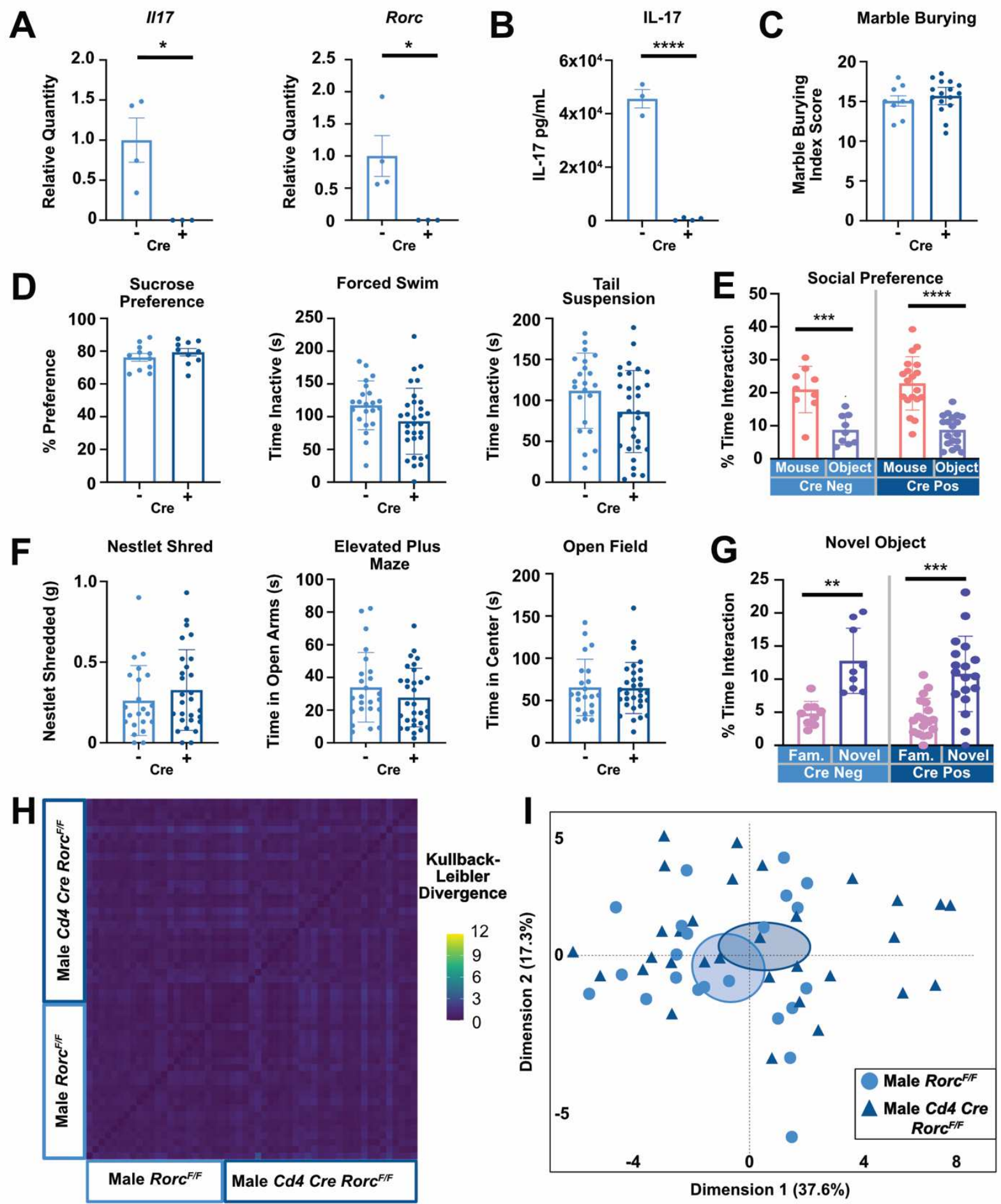

Figure 3: Depletion of Rorc does not Induce Spontaneous Behavioral Changes in Male Mice. Loss of gene expression of both //17 and Rorc in in vitro skewed Th17s from Rorc KO mice and littermate controls by qPCR (A). T test, $n=3$ /group. ELISA representing loss of IL-17 in in vitro skewed Th17s from Rorc KO animals (B). T test, $n=3$ or 4/group. Baseline marble burying (C), depressive-like (D), social preference (E), anxiety-like (F), and novel object recognition (G) 
behaviors in Rorc KO mice vs littermate. T tests. Kullback-Leibler divergence heatmap $(\mathrm{H})$ and PCA (I) plot of DeepLabCut analyzed behaviors between groups. Each dot represents an animal, $\mathrm{N}=2$, male mice. 

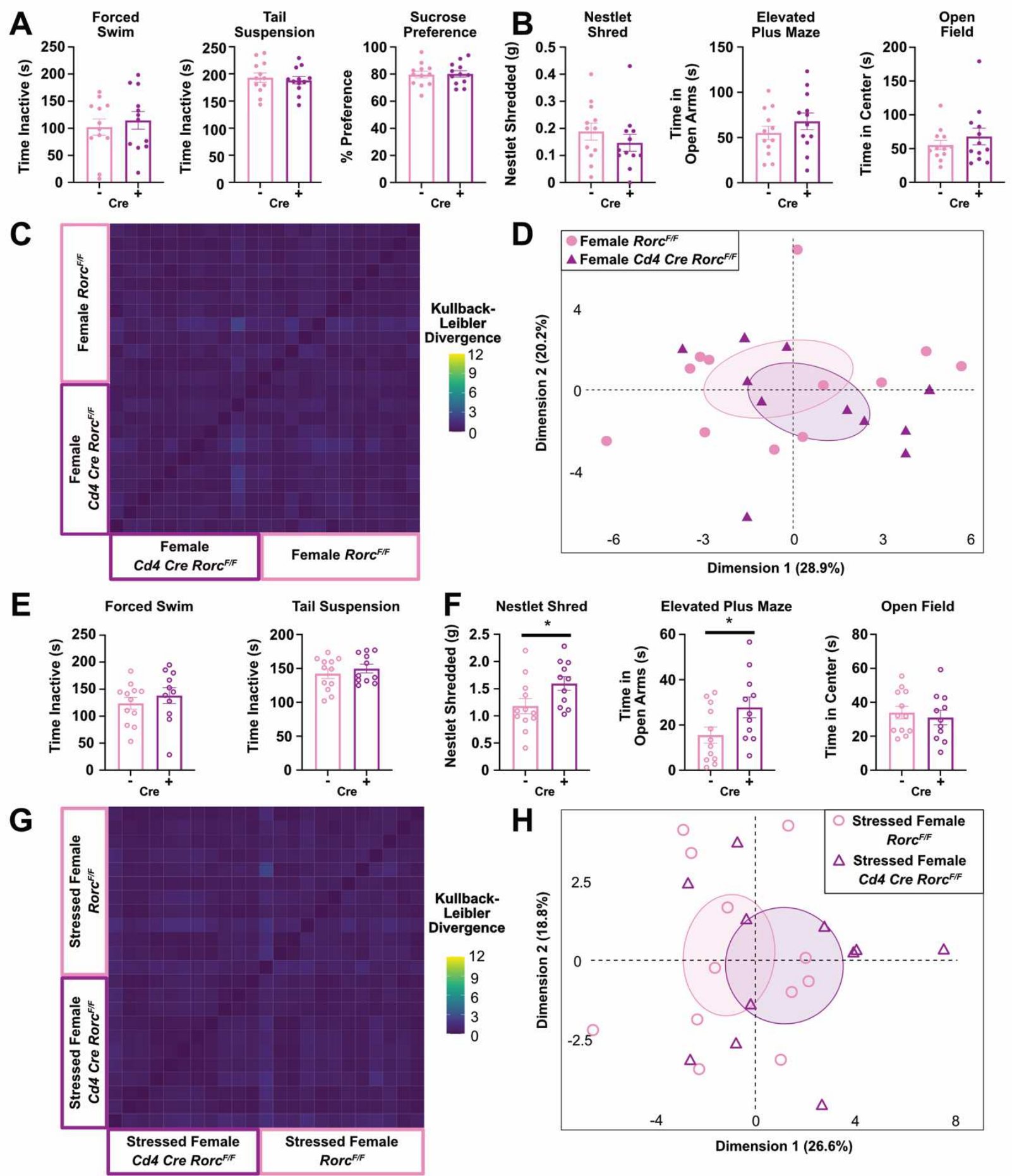

Figure 4: Loss of Rorc in T cells does not Impact Depressive- or Anxiety- like Behaviors in Female Mice. No differences in depressive-like (A) or anxiety-like (B) behaviors between female Rorc KO and littermate controls at baseline. No differences in the Kullback-Leibler divergence heatmap (C) or PCA (D) plot of DeepLabCut analyzed baseline behaviors between groups. Depressive-like (E) and anxiety-like (F) behaviors after 3 weeks of UCMRS between female Rorc $\mathrm{KO}$ and littermate controls. Kullback-Leibler divergence heatmap (G) and PCA (H) plot of 
DeepLabCut analyzed behaviors after 3 weeks of UCMRS between groups. T tests, each dot represents an animal, $\mathrm{N}=1$, female mice. 


\section{Supplementary Files}

This is a list of supplementary files associated with this preprint. Click to download.

- supplementarymaterial.pdf 